\title{
Efficacy of Neuromodulation Therapy with Vagus Nerve Stimulator in Patients with Drug-Resistant Epilepsy on Unchanged Antiepileptic Medication Regimen for 24 Months Following the Implant
}

Sirichai Chayasirisobhon*, Leslie Cahan, SooHo Choi, Bruce Enos, Jane Hwang, Meei Lin, Erika Pietzsch, Jeffrey Schweitzer, Benjamin Spurgeon and Suresh Gurbani

Comprehensive Epilepsy Program, Southern California Permanente Medical Group, California, USA

*Corresponding author: SirichaiChayasirisobhon, Department of Neurology, Kaiser Permanente Medical Center, 3460 E. La Palma Avenue, Anaheim, CA, 92806, USA, Tel: 7142968147; Fax: 7146442274 E-mail: siri.chayasirisobhon@kp.org

Received date: Nov 14, 2014, Accepted date: Feb 06, 2015, Published date: Feb 12, 2015

Copyright: $\odot 2015$ Chayasirisobhon S, et al. This is an open-access article distributed under the terms of the Creative Commons Attribution License, which permits unrestricted use, distribution, and reproduction in any medium, provided the original author and source are credited.

\begin{abstract}
To establish efficacy of vagus nerve stimulation (VNS) therapy in the treatment of intractable epilepsy, we compared outcome data from the baseline period to the maintenance period ( 7 to 24 months post-implant) by calculating the mean seizure frequency at 6-month intervals ( 7 to 12 months - first study period, 13 to 18 months second study period, and 19 to 24 months - third study period), in 39 consecutive patients on unchanged antiepilepsy drug (AED) regimen for 24 months following the VNS implant. Of the 39 patients24 (61.5\%) at first study period, $25(64.1 \%)$ at second study period and $25(64.1 \%)$ at third study period were responders ( $\geq 50 \%$ reduction in seizures). Twenty one (53.9\%) patients were responders in all three study periods. Incremental seizure control was seen in 15 of these 21 patients. Although $3(7.7 \%), 4(10.3 \%)$ and $8(20.5 \%)$ patients had a total (100\%) seizure control at first, second and third study periods respectively, no patient remained seizure-free through all 3 study periods. Seven (17.9\%) patients were partial responders ( $\geq 50 \%$ seizure reduction in two or less study periods). Eleven patients $(28.2 \%)$ were non-responders $(<50 \%$ seizure reduction in all 3 study periods). Twenty three patients (59\%) had partial epilepsy with and without secondarily generalization and 16 patients (41\%) had primary generalized epilepsy. Eleven $(47.8 \%)$ of the 23 patients with partial epilepsy and $10(62.5 \%)$ of the 16 patients with generalized epilepsy were responders in all three study periods. We conclude that: 1) More than $60 \%$ of patients on unchanged AED regimen continued to be responders at 24 months following the VNS implant. 2) A trend towards increasing responder rate with increasing duration of VNS therapy was observed.3) No major complications or side effects requiring discontinuation of VNS therapy were noted.
\end{abstract}

Keywords: Drug resistant epilepsy; Vagus nerve stimulation

\section{Introduction}

Vagus nerve stimulation (VNS) therapy is an effective adjunctive neuromodulation treatment for medically refractory epilepsy [1-5]. Several studies that demonstrated VNS efficacy have reported $\geq 50 \%$ seizure reduction in $23.4 \%-39 \%$ patients with follow up duration of 3-3.5 months [5-8],35\% - 61.8\% with follow up duration of 6-12 months [9-12] and 39\% - 63.8\% with follow up duration of 1-10 years [13-25]. However, as antiepileptic drug (AED) regimens during the study period were adjusted as needed, these studies do not truly reflect the role of VNS in incremental seizure frequency reduction seen with longer duration of VNS therapy. So far only two studies (with followup for 12 months and 18 months respectively) have demonstrated the continued efficacy of VNS with stable AED regimens $[18,26]$. We present the results of a retrospective analysis study of prospectively collected data showing the efficacy and safety of VNS therapy in a group of patients with intractable epilepsy in whom the AED regimen remained unchanged(due to either non-availability of next generation AEDs or patients' refusal to change AED regimen) for a period of 24 months following VNS implant.

\section{Methods}

All the patients who undergo treatment at our Comprehensive Epilepsy Center maintain a seizure diary which is entered in the data base during follow up visits on an ongoing basis. We conducted a retrospective analysis of this prospectively collected clinical data of 160 consecutive patients with drug resistant epilepsy (failed adequate trial of at least 3 appropriate AEDs)who underwent vagus nerve stimulator (VNS) implantation surgery at Kaiser Permanente Medical Centers in Anaheim and Los Angeles in California, U.S.A. from September 1998 to December 2011. A total of 39 patients were identified in whom AED regimen had remained unchanged for a period of 24 months following VNS implant. The patients were on high therapeutic doses of AEDs, which remained unchanged either due to non-availability of next generation AEDs or patients' refusal to change AED regimen.

All patients underwent pre-operative evaluation which included long term video EEG monitoring, MRI brain examination and approval by Kaiser multidisciplinary epilepsy surgery case conference. Patients were admitted for surgical implantation of VNS device from Cyberonics by our neurosurgeons, who had received required training.

To allow wound healing, the VNS system was not activated for 1 week postoperatively. With starting current being $0.25 \mathrm{~mA}$, output current was gradually increased in $0.25 \mathrm{~mA}$ increments once per week for six weeks and subsequently during biweekly visits up to maximum of $3.5 \mathrm{~mA}$ if needed. Output current was adjusted on the basis of 
Citation: Chayasirisobhon S, Cahan L, Choi SH, Enos B, Hwang J, et al. (2015) Efficacy of Neuromodulation Therapy with Vagus Nerve Stimulator in Patients with Drug-Resistant Epilepsy on Unchanged Antiepileptic Medication Regimen for 24 Months Following the Implant. J Neurol Neurophysiol 6: 268. doi:10.4172/2155-9562.1000268

Page 2 of 4

patients' tolerance to the electrical stimulation and impact on seizure control. The VNS stimulation parameters were either standard cycle or rapid cycle per preference of the treating epileptologist. For standard cycling, the signal-on time was $>30$ seconds and signal-off time ranged from 3 minutes to 5 minutes. For rapid cycling, signal-on time was $<$ 21 seconds and signal-off time ranged from 0.2 minutes to 1.8 minutes. Duty cycle was maintained at $<50 \%$ in most cases.

The first 6 months after VNS implant was the titration period and the period from 6 months to 24 months was the study period. Efficacy of VNS therapy was analyzed by calculating mean change in seizure frequency from baseline (seizures frequency in the last 6 months before patients received VNS implant) to mean seizure frequency for 6-month intervals for the three study periods (7 to 12 months - first study period, 13 to 18 months - second study period, and 19 to 24 months - third study period). We determined the VNS therapy success rate by calculating reduction of seizure frequency in different types of epilepsies during the three study periods. Those with $\geq 50 \%$ reduction of seizure frequency in all three study periods with respect to the preimplant seizure frequency were defined as total responders; those with $\geq 50 \%$ reduction of seizure frequency in less than three study periods were categorized as partial responders; and those with $<50 \%$ reduction of seizure frequency in all three study periods as nonresponders.

We also assessed postoperative adverse events, side effects, and tolerability of the implantation procedure and of the VNS device, and impact on alertness, mood and behavior.

\section{Results}

There were 20 females and 19 males with age ranging from 5 years to 70 years. The mean age at onset of epilepsy was $8.8 \pm 11.9$ years and mean age at VNS implantation was $21.3 \pm 14.6$ years. Mean number of AEDs at baseline was 2.02. Clinical characteristics of the patients at base line are summarized in Table 1.

\begin{tabular}{|l|l|}
\hline Etiology & Number of Patients \\
\hline Lennox Gastaut syndrome & 6 \\
\hline Encephalitis & 4 \\
\hline Tuberous sclerosis & 3 \\
\hline Tumor & 3 \\
\hline Head injury & 2 \\
\hline Porencephaly & 1 \\
\hline Prenatal encephalopathy & 1 \\
\hline Unknown & 19 \\
\hline Antiepileptic drugs & 2 \\
\hline 1 drug & 8 \\
\hline 2 drugs & 23 \\
\hline 3 drugs & 7 \\
\hline 4 drugs & 1 \\
\hline Epilepsy Type & 2 \\
\hline Simple partial & \\
\hline
\end{tabular}

\begin{tabular}{|l|l|}
\hline Complex partial & 13 \\
\hline $\begin{array}{l}\text { Complex partial with secondary } \\
\text { generalization }\end{array}$ & 8 \\
\hline Generalized tonic-clonic & 3 \\
\hline Atypical absence & 4 \\
\hline Tonic & 2 \\
\hline Mixed generalized & 7 \\
\hline
\end{tabular}

Table 1: Clinical characteristics of the patients at base line

In 24 month period, 34 patients were treated with the standard cycle of VNS. Four patients who initially were on standard cycling were later switched to rapid cycling. One patient was treated with only rapid cycling. The output current used ranged from $0.5 \mathrm{~mA}$ to $3.5 \mathrm{~mA}$ with median setting of $2.5 \pm 0.7 \mathrm{~mA}$ at 6 months, $2.8 \pm 0.7 \mathrm{~mA}$ at 12 months, and $2.8 \pm 0.7 \mathrm{~mA}$ at 24 months.

Responder rates during the three study periodsare shown in Table 2. Twenty one patients (53.9\%) had $\geq 50 \%$ reduction in seizure frequency in all three study periods, 15 of which showed incremental response. Although $3(7.7 \%), 4(10.3 \%)$ and $8(20.5 \%)$ patients had a total $(100 \%)$ seizure control at first, second and third study periods respectively, no patient remained seizure-free through all 3 study periods. Partial response was seen in $7(17.9 \%)$ patients. Eleven patients $(28.2 \%)$ were non-responders or had worsening of seizure frequency.

Of the 39 patients, $\geq 50 \%$ reduction in seizure frequency was seen in 15 patients (38.5\%) during the titration period, in 24 patients $(61.5 \%)$ during the first study period (7 to 12 months), in 25 patients $(64.1 \%)$ during the second period (13to 18 months) and in 25 patients $(64.1 \%)$ during the third period (19 to 24 months) (Figure 1).

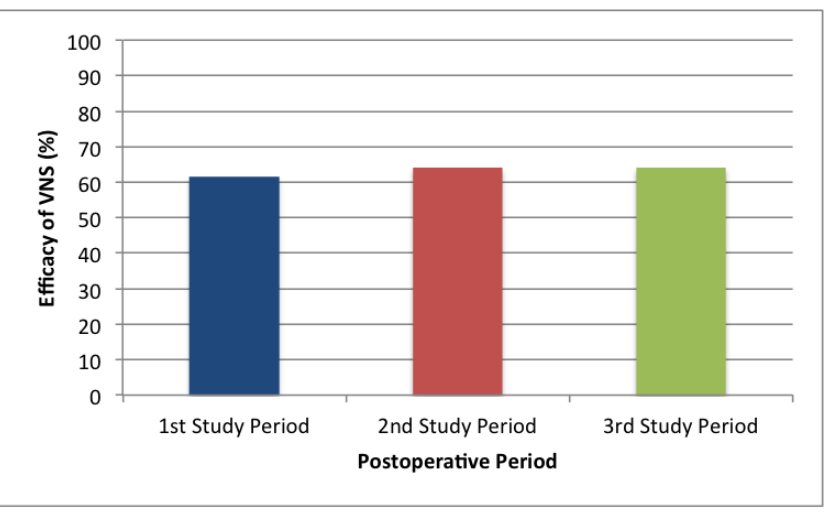

Figure 1: Efficacy of VNS ( $\geq 50 \%$ reduction in seizure frequency) in all 3 Study periods

We also analyzed the efficacy of VNS according to the type of epilepsy. Out of 39 patients, 23 (59\%) had partial epilepsy with and without secondary generalization, and $16(41 \%)$ had primary generalized epilepsy. Eleven (47.8\%) of the 23 patients with partial epilepsy and $10(62.5 \%)$ of the 16 patients with generalized epilepsy showed $\geq 50 \%$ reduction of seizure frequency. 
Citation: Chayasirisobhon S, Cahan L, Choi SH, Enos B, Hwang J, et al. (2015) Efficacy of Neuromodulation Therapy with Vagus Nerve Stimulator in Patients with Drug-Resistant Epilepsy on Unchanged Antiepileptic Medication Regimen for 24 Months Following the Implant. J Neurol Neurophysiol 6: 268. doi:10.4172/2155-9562.1000268

Page 3 of 4

Twenty three patients (59\%) were reported being more alert over the 24 months study period. Twenty two (56.4\%) patients experienced intermittent hoarseness, cough and/or throat irritation when signal was "on". These side effects subsided after the output current parameters were lowered and therefore did not necessitate discontinuation of the VNS treatment in any patient.

Vital signs and electrocardiographic data analysis showed no clinically significant change while on VNS therapy.

\section{Discussion}

VNS therapy has been established as an effective therapy for drugresistant epilepsy [1-5]. No particular AED has been identified as having additive anti-seizure effect with VNS therapy. If the net effectiveness of VNS therapy is to be established, it is imperative to maintain the doses of concomitant AEDs constant. Many studies on VNS therapy have reported the incremental increase in responder rate with increasing duration of treatment. However, the success rate reported in most of these long term studies does not adequately reflect the effectiveness of VNS therapy as AEDs were not kept constant during the study period. Frequent follow up visits required during early VNS ramp-up stage, which facilitate careful adjustment of AED regimen, and the potential for addition of newer generation AEDs permitted during the study period, may have contributed to the reported reduction in seizure frequency [12-25]. On the other hand, the patients' and the treating neurologists' desire to achieve decreased dose and reduced number of AEDs after VNS implantation may have resulted in the reported impact of VNS therapy being suboptimal.

A 2002 study involving 1407 patients who were treated with VNS therapy for at least 12 months reported no difference in VNS efficacy between patients on stable regimen and those with AED adjustment [12]. This result probably indicates either ability to optimize and thus achieve better efficiency of VNS therapy due to stable AED pharmacodynamics and/or lack of improved efficacy due to maximum response to AEDs already attained. Interestingly, the same study also showed that $40 \%$ to $50 \%$ of patients on VNS therapy were able to reduce the dose of concomitant AEDs without adversely affecting seizure control and at the same time achieving improved quality of life [12]. As no specific protocol was followed while adjusting AED regimen, the results of this study should be reviewed with caution.

To date, only two studies where AED regimen was kept constant (with follow-up for 12 months and 18 months respectively) have demonstrated continued efficacy of VNS therapy $[18,26]$. The first study $(n=269)$ demonstrated $\geq 50 \%$ reduction of seizure frequency in $57 \%$ of the patients after 12 month of therapy following VNS implant [18]. A more recent study $(n=43)$ demonstrated that $62.8 \%$ patients had $\geq 50 \%$ reduction in seizure frequency at 18 months follow-up [26]. In our study $(\mathrm{n}=39)$, the efficacy of VNS is analyzed by calculating the mean seizure frequency at 6-month intervals from the baseline to 24 month period. The response of VNS therapy is demonstrated throughout the 24-month period. Results of our study and 2 other similar studies where AED regimens were kept constant following VNS implant fall within the higher range of the VNS efficacy (35\% to $61.8 \%$ ) reported by other long term follow up (1-10 years) studies where modification of concomitant AEDs was permitted [9-12], thereby proving the efficacy of VNS therapy.

We also demonstrate a trend towards increasing responder rate with increasing duration of VNS therapy (Table 2).

\begin{tabular}{|l|l|}
\hline Study Period & Number of patients \\
\hline Total Responders & 21 \\
\hline Partial Responders & \\
\hline Period from 7 months to 12 months & 1 \\
\hline Period from 7 months to 18 months & 2 \\
\hline Period from 13 months to 24 months & 4 \\
\hline Non- responders & 11 \\
\hline
\end{tabular}

Total Responders: $\geq 50 \%$ reduction of seizure frequency in all three study periods; Partial Responders: $\geq 50 \%$ reduction of seizure frequency in $<$ three study periods; Non-responders: $<50 \%$ reduction of seizure frequency in all three study periods.

Table 2: Responder rate ( $\geq 50 \%$ reduction of seizure) at 3 study periods

In our study, VNS appears to have the antiepileptic effects on both partial and primary generalized epilepsies with responder rates of $47.8 \%$ and $62.5 \%$ respectively. Our previous study [11] also demonstrated that patients with primary generalized epilepsy had better responder rate than patients with partial epilepsy with and without generalized epilepsy. However, small study size may precludedelineating statistical significance of these numbers.

Cough and pharyngeal pain commonly occur during initial application of current or when incremental increases of output current are too large. These adverse events can be minimized by increasing the current at $0.25 \mathrm{~mA}$ increments or setting the pulse width to the lower value. Voice alteration such as hoarseness which occurs in many patients during the stimulation resolves over time and therefore may not require any lowering of output current setting. No major complications or side effects requiring discontinuation of VNS therapy were noted in our study.

\section{Conclusion}

We conclude that:

1) More than $60 \%$ of patients on unchanged AED regimen continued to be responders at 24 months following the VNS implant.

2) A trend towards increasing responder rate with increasing duration of VNS therapy was observed.

3) No major complications or side effects requiring discontinuation of VNS therapy were noted.

We realize the limitations of the small size of our retrospective data analysis study but still consider it to be an important study as 1) due to ethical issues it is not possible to design a prospective double blind large research study to further characterize the findings of this study; and 2) due to availability of third-generation AEDS, in the near future similar retrospective data analysis study will not be available.

\section{References}

1. The Vagus Nerve Stimulation Study Group (1995) A randomized controlled trial of chronic vagus nerve stimulation for treatment of medically intractable seizures. Neurology 45: 224-230.

2. Salinsky MC, Uthman BM, Ristanovic RK, Wernicke JF, Tarver WB (1996) Vagus nerve stimulation for the treatment of medically intractable 
Citation: Chayasirisobhon S, Cahan L, Choi SH, Enos B, Hwang J, et al. (2015) Efficacy of Neuromodulation Therapy with Vagus Nerve Stimulator in Patients with Drug-Resistant Epilepsy on Unchanged Antiepileptic Medication Regimen for 24 Months Following the Implant. J Neurol Neurophysiol 6: 268. doi:10.4172/2155-9562.1000268

Page 4 of 4

seizures: results of a 1-year open-extension trial. Vagus Nerve Stimulation Study Group. ArchNeurol 53: 1176-1180.

3. Hornig GW, Murphy JV, Schallert G, Tilton C (1997) Left vagus nerve stimulation in children with refractory epilepsy: an update. South Med J 90: 484-488.

4. Murphy JV, Hornig G, Schallert G (1995) Left vagal nerve stimulation in children with refractory epilepsy: preliminary observations. Arch Neurol 52: 886-889.

5. Ben-Menachem E, Mañon-Espaillat R, Ristanovic R, Wilder BJ, Stefan H, et al. (1994) Vagus nerve stimulation for treatment of partial seizures: 1 . A controlled study of effect on seizures. First International Vagus Nerve Stimulation Study Group. Epilepsia 35: 616-626.

6. George R (1995) A randomized controlled trial of chronic vagus nerve stimulation for treatment of medically intractable seizures. Neurology 45 : 224-230.

7. Handforth A, DeGiorgio CM, Schachter SC, Uthman BM, Naritoku DK, et al. (1998) Vagus nerve stimulation therapy for partial-onset seizures: a randomized active-control trial.Neurology 51: 48-55.

8. DeGiorgio C, Heck C, Bunch S, Britton J, Green P, et al. (2005) Vagus nerve stimulation for epilepsy: randomized comparison of three stimulation paradigms. Neurology 65: 317-319.

9. DeGiorgio CM, Schachter SC, Handforth A, Salinsky M, Thompson J, et al. (2000) Prospective long-termstudy of vagus nerve stimulation for the treatment of refractory seizure. Epilepsia 41:1195-1220.

10. Frost M, Gate J, Helmers SL, Wheless JW, Levisohn P, et al. (2001) Vagus nerve stimulation in children with refractory seizures associated with Lennox Gastaut syndrome. Epilepsia 42: 1148-1152.

11. Chayasirisobhon S, Chayasirisobhon WV, Koulouris S, Gurbani S (2003) Vagus Nerve Stimulation in Drug-Resistant Epilepsy. ActaNeurol Taiwan 12: 123-129.

12. Labar DR (2002) Antiepileptic drug use during the first 12 months of Vagal nerve stimulation therapy: a registry study. Neurology 59: S38-S43.

13. Amar AP, DeGiorgio CM, Tarver WB, Apuzzo ML (1999) Long-term multi-center experience with vagus nerve stimulation for intractable partial seizure:results of the XE5 trial. Steriotact Funct Neurosurg 73: 104-108.

14. Ben-Menachem E, Hellstrom K, Waldton C, Augustinsson LE, et al. (1999) Evaluation of refractory epilepsy treated with vagus nerve stimulation for up to 5 years.Neurology 52: 1265-1267.

15. Scherrmann J, Hoppe C, Kral T, Schramm J, Elger CE, et el. (2001) Vagus nerve stimulation: clinical experience in a large patient series. J clinneurophysiol 18: 408-414.
16. Murphy JV, Torkelson R, Dowler I, Simon S, Hudson S (2003) Vagal nerve stimulation in refractory epilepsy: the first You +1 'd this publicly. Undo100 patients receiving vagus nerve stimulationat a pediatric epilepsy center. ArchPediatrand Adolesc Medicine 157: 560-564.

17. Amar AP, Apuzzo ML, Liu CY (2008) Vagus nerve stimulation therapy after failed cranial surgery for intractable epilepsy: results from the vagus nerve stimulation therapy patient outcome registry. Neurosurgery 62 : 1086-1093.

18. Labar D (2004) Vagus nerve stimulation for 1 year in 269 patients on unchanged antiepileptic drug.Seizure 13: 392-398.

19. Uthman BM, Reichl AM, Dean C, Eisenschenk S, GilmoreR, et al. (2004) Effectiveness of vagus nerve stimulation in epilepsy patients: a 12-year observation. Neurology 63: 1124-1126.

20. Vonck K, Thadani V, Gilbert K, Dedeurwaerdere S, De Groote L, et al. (2004) Vagus nerve stimulation for refractory epilepsy: a transatlantic experience. JClinNeurophysiol 21: 283-289.

21. Alexopoulos AV, Kotagal P, Loddenkemper T, Hammel J, Bingaman WE (2006) Long-term results with vagus nerve stimulation in children with pharmacoresistant epilepsy. Seizure 15: 491-503.

22. Benifla M, Rutka JT, Logan W, Donner EJ (2006) Vagal nerve stimulation in refractory epilepsy in children: Indication and experience atthe hospital for sick children. Childs NervSyst 22: 1018-1026.

23. De Herdt V, Boon P, Ceulemans B, Hauman H, Lagae L, et al. (2007) Vagal nerve stimulation for refractory epilepsy: a Belgian multi-center study. Eur JPaediatrNeurol 11:261-269.

24. Elliott RE, Morsi A, Kalhorn S, Marcus J, Sellin J, Kang M, et al. (2011) Vagus nerve stimulation in 436 consecutive patients with treatmentresistant epilepsy: Long-term outcomes and predictors of response. Epilepsy Behav 20: 57-63.

25. Elliott RE, Morsi A, Tanweer O, Grobelny B, Geller E, Carlson C, et al. (2011) Efficacy of vagus nerve stimulation over time: review of 65 consecutive patients with treatment-resistant epilepsy treated with VNS > 10 years. Epilepsy Behav 20: 478-483.

26. Garcia-Navarrete E, Torres C, Gallego I, Nava M, Pastor J, et al. (2013) Long-term results of vagus nerve stimulation for adults with medicationresistant epilepsy who have been on unchanged antiepileptic medication. Seizure 22: 9-13. 